\title{
Minireview
}

\section{Application of the New Logistic Model to Microbial Growth Prediction in Food}

\author{
HIROSHI FUJIKAWA \\ Laboratory of Veterinary Public Health, Faculty of Agriculture, \\ Tokyo University of Agriculture and Technology \\ 3-5-8 Saiwai-cho, Fuchu, Tokyo 183-8509, Japan
}

Received 3 February, 2011/Accepted 9 April, 2011

\begin{abstract}
Recently a microbial growth model, the new logistic model, which could precisely describe and predict microbial growth at various patterns of temperature, was developed by the author (Biocontrol Science, 15, 75-80, 2010). The author shows several software programs developed with the model in this review. First, a program that analyzes microbial growth data and generates growth curves fitted to the model was developed. Second, a growth prediction program for Escherichia coli, Staphylococcal aureus, and Vibrio paraheamoliticus exposed at various patterns of temperature was made based on experimental data. For $V$. paraheamoliticus a program for bacterial growth under environmental conditions including temperature, salt concentration, and $\mathrm{pH}$ was developed. These programs are available free at the Japan Food Industry Center. Furthermore, a method to estimate the temperature at various points on or inside a food exposed to a given temperature was developed by using the measured temperatures of two points on the surface of the food and the heat conduction law. Combining this method with the growth model, a system that predicts microbial growth in a food exposed to various temperature patterns was made. This system could be a prototype of an alert system for microbial food safety.
\end{abstract}

Key words : Heat transfer/Logistic model/Predictive model/Microbial growth/Alert system

\section{INTRODUCTION}

For the last two decades, a number of mathematical models have been developed to quantitatively describe microbial growth in culture media and food (McMeekin et al., 1993). The field of such studies has been called predictive food microbiology; the goal is to predict microbial growth and death in food with mathematical models based on the environmental factors such as temperature, the atmosphere (oxygen level), nutrition level, the hydrogen ion concentration $(\mathrm{pH})$, salt and/or sugar concentrations, and water activity of the food. If microbial growth in a given food can be predicted with the models from those environmental factors, it would be a great help

${ }^{*}$ Corresponding author. Tel/Fax : +81-42-367-5937, E-mail : fujik (a)cc.tuat.ac.jp to assess the microbial food safety of the food before it reaches the consumers.

There have been some mathematical models that are well known worldwide, including the modified Gompertz model, Baranyi model, and a three-phase linear model (Gibson et al., 1987; Baranyi and Roberts, 1995; Buchanan, 1997). However, they have some theoretical and practical problems (Fujikawa, 2010). The author and his colleagues recently developed a model, which is an extended form of the logistic model for the description of microbial growth (Fujikawa et al., 2003; Fujikawa and Morozumi, 2005). Here we call it the new logistic model as shown in Eq. (1).

$$
\frac{d N}{d t}=r N\left\{1-\left(\frac{N}{N_{\max }}\right)^{m}\right\}\left\{1-\left(\frac{N_{\min }}{N}\right)^{n}\right\}
$$

Here $N$ is the population of the microorganism $(\mathrm{CFU} / \mathrm{g}$ or $\mathrm{CFU} / \mathrm{ml}$ ) at time $t(\mathrm{~h}), r$ is the rate 
constant of growth $(1 / \mathrm{h})$, and $N_{\max }$ is the maximum population (CFU/g or CFU $/ \mathrm{ml}$ ). $N_{\text {min }}$, is the minimum population related to the initial population (CFU/g or $\mathrm{CFU} / \mathrm{ml}) . m$ and $n$ are parameters.

In a recent review (Fujikawa, 2010), the author described the history of model development, the characteristics of the model, and the performance of the model in predicting microbial growth and microbial toxin production at dynamic temperatures. In the present review, the author further shows how the new logistic model has been applied to microbial food safety. Namely, the author here explains computer programs for fitting microbial data to the model and for predicting microbial growth, and an alert system for microbial hazards which is made with the growth model and a temperature estimation method for food.

\section{Development of a Program to Fit Data to the new Logistic Model for Microbial Growth.}

Researchers on food microbiology have a lot of microbial growth data in food and/or media of their own. Those data should be analyzed quantitatively with growth models to use it for food safety. Baranyi et al. have provided a free software called DMFit, where data are analyzed with the Baranyi model (http://www.ifr.ac.uk/safety/dmfit/). The author thus developed a computer program to fit growth data to the new logistic model on a spread sheet program, Microsoft Excel 2003, the most commonly used calculation program worldwide (Fujikawa and Kano, 2009a). The fitting is done to minimize the mean of squared errors, MSE, between the measured and estimated cell concentrations (in log units) during the observation period. The program has been also developed so that it does not require any special knowledge about the mathematical model and computer program.

The procedure for the data fitting program is as follows (Fig. 1) (Fujikawa and Kano, 2009a). First, users need to input raw growth data that consist of a set of storage periods (in hours) and cell numbers (in log units). To do this, the fitting program has two buttons. That is, by clicking on the "Delete Data" button, all data that users have previously input are removed. After copying a new data set, users can paste it in with the "Paste Data" button. Second, the "Curve Fitting" button is clicked to fit and analyze the data. Then a window that requires the slope position to be input in a growth curve will appear on the screen. A growth curve is commonly a sigmoidal one with a slope, or the logarithmic phase. For the curve fitting, the position of the slope is essentially important. Thus, the candidates for the starting point and ending point of the slope are input on the screen. When the

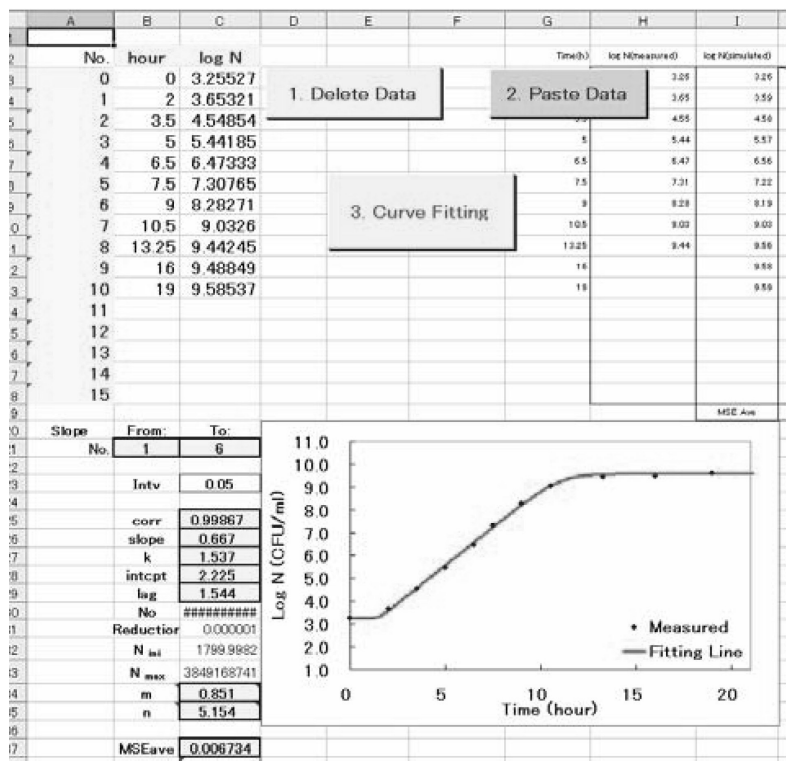

FIG. 1. Data fitting program with the new logistic model. The best-fit curve and its characteristics are shown on the screen.

"OK" button is clicked, the program starts fitting the data. With each combination of the starting and ending points, a curve fitted to the growth data is generated. Results for all combinations are shown in a separate sheet. The value of MSE between the measured and estimated cell concentration (in log units) during the observation period is given for each fitted curve. Among the curves, the curve with the smallest MSE is chosen as the best-fitted curve. The best-fit curve then appears on the screen of the fitting program (Fig. 1). The characteristics of the curve such as the correlation coefficient of the slope, the rate constant of growth $(r)$, the duration of the lag period, and the value of MSE for the observation period are also shown.

The program can show good fitting performance, as seen in Fig. 1. Thus, program users easily can get a growth curve fitted to their raw data. Furthermore, the obtained analysis can be used for the prediction of microbial growth at changing temperatures. These could be useful in quantitatively studying microbial growth in food products and then lead to developing a risk analysis for microbial food poisoning outbreaks and food spoilage. The program is provided free by the author.

\section{Development of a predictive program for mi- crobial growth under various temperature conditions.}

As stated above, the goal of the predictive growth models is to predict microbial growth in food from the environmental conditions. There are some inter- 
nationally well-known computer programs for microbial growth and death such as the pathogen modeling program (PMP) by the U. S. Department of Agriculture (http://ars.usda.gov/Service/). However, PMP can predict microbial growth at constant temperatures only. Recently, Combase (http:// wyndmoor.arserrc.gov.combase/), an international database for microbial growth and death, was developed. The data in Combase are collected from papers published in international journals on food microbiology, but some of the collected data are not complete, resulting in poor predictions.

The author also worked to develop programs for predicting microbial growth in food with the new logistic model for domestic food industries in Japan with a support of the Japan Food Industry Center. The programs have been developed on Microsoft Excel 2003. The program predicts Escherichia coli growth in broth, Staphylococcus aureus growth and its enterotoxin production in milk, and Vibrio parahaemolyticus growth in broth at various temperature patterns. Experimental data for prediction were obtained from studies by the author and his colleagues (for E. coli and S. aureus) and by Dr. Fujii et al. (for V. parahaemolyticus) (Fujikawa et al., 2006).

For growth prediction, the value for $r$ in Eq. (1) is estimated from temperature, $T$, at time $t$ during the growth with the Arrhenius model or the square root model. Examples of the two models are respectively shown below.

$$
\text { 1n } r=21.0-6230 / T
$$

$$
\sqrt{r}=0.0452(T-1.93)
$$

Here $T$ in Eq. (2) is the absolute temperature (K). For $m$ and $n$ in Eq. (1), the averages of observed values for the microorganisms at various constant temperatures are input.

For $S$. aureus, the program can predict a growth curve as well as the amount of staphylococcal enterotoxin A. Bacterial growth was predicted with by the new logistic model. Procedures for toxin production prediction were described in an earlier paper (Fujikawa and Morozumi, 2006).

The program is user-friendly; users can easily input the temperature history of a test food and obtain the prediction instantly on the computer screen. The procedures for the prediction are demonstrated in Fig. 2. (Fujikawa et al., 2006). When users have a sequential data set of temperature during the observation period in an Excel file, they can also get information on the prediction by pasting the data set on the

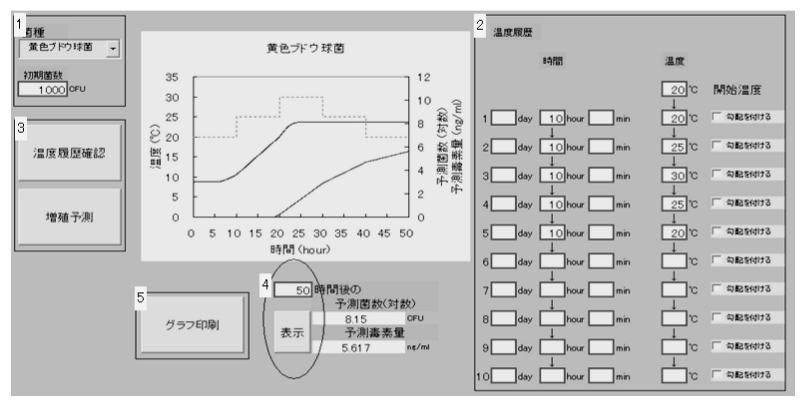

FIG. 2. Microbial growth prediction program. Circled numbers show the order of procedure. 1, Input of microbial species and initial cell population (CFU); 2, Input of the temperature history; 3 , confirmation of the temperature history and implementation of growth prediction; 4, Estimation of cell population ( $\log \mathrm{CFU}$ ) and staphylococcal enterotoxin A concentration in milk ( $\mathrm{ng} / \mathrm{ml}$ ) for the storage period of interest; 5 , printing of the graph.

prediction program.

For $V$. parahaemolyticus, a new growth prediction program was further developed (Fujikawa, et al., 2009). Namely, environmental factors other than temperature were also included for prediction. The factors are salt concentration and $\mathrm{pH}$. An O3:K6 strain, V03-80 was used as a test strain for this study (Kimura et al., 2008). The strain was selected among a total of $34 \mathrm{~V}$. parahaemolyticus strains studied, because it is one of the strains that grew well at high salt concentrations. Values of parameters $r, m$, and $n$ of the new logistic model were described with a polynomial model. An example for $r$ with a 3-order polynomial equation is shown as follows:

$$
\begin{aligned}
r= & a_{1} T^{3}+a_{2} S^{3}+a_{3} P^{3}+a_{4} T^{2} S+a_{5} S^{2} P+a_{6} P^{2} T \\
& +a_{7} T^{2} P+a_{8} S^{2} T+a_{9} P^{2} S+a_{10} S P+a_{11} T^{2}+a_{12} S^{2} \\
& +a_{13} P^{2}+a_{14} T S+a_{15} S P+a_{16} P T+a_{17} T+a_{18} S \\
& +a_{19} P+a_{20}
\end{aligned}
$$

where $T$ is temperature $\left({ }^{\circ} \mathrm{C}\right), S$ is the salt concentration of the broth (\%), and $P$ is $\mathrm{pH}$ of the broth. $a_{1}$, $a_{2}, \cdots, a_{20}$ are the parameters of the polynomial equation. The optimal values for the parameters $a_{1}$ to $a_{20}$ were estimated by the least square method between the observed and estimated values of $r$ with the Solver in Microsoft Excel 2003. Thus, users can get the best value for $r$ at certain values for temperature, the salt concentration, and $\mathrm{pH}$. The same procedures are done for $m$ and $n$. Finally, by inputting the environmental conditions of temperature, $\mathrm{pH}$, and salt concentration in this program, they can instantly get a predicted growth curve of the organism (Fujikawa, et al., 2009) (Fig. 3).

These software programs shown in Figs. 2 and 3 are provided free by the Japan Food Industry Center 


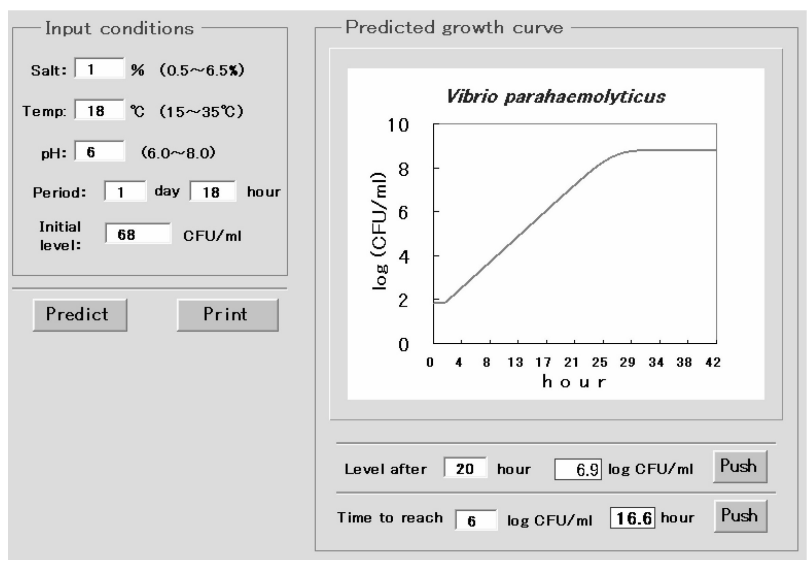

FIG. 3. V. parahaemoliticus predictive growth program. After the environmental conditions on salt concentration, temperature, $\mathrm{pH}$, the period of storage, and the initial bacterial level are input in the upper left boxes, a predicted growth curve is generated in a graph. The bacterial level at the time of concern and the time to reach the bacterial level of concern are also predicted.

(http://www.shokusan.or.jp/haccp/news/index_18. $\mathrm{html})$. The growth and toxin production predicted can be important indices to determine whether the food of concern is microbiologically safe or not. As a result, the program can be also used for the evaluation of exposure assessment in risk analysis for the food of interest. On the other hand, the data for prediction come from full nutrient conditions without any microbial competition. Thus, users should know that the predicted growth shows the worst scenario from the viewpoint of food safety under the given environmental conditions, which means that the predicted curves would be the best ones for the microbes.

\section{Simulation of Microbial Growth in Food us- ing its Estimated Temperatures}

\section{A. Estimation of temperatures in a food from the surface temperature.}

The temperature of a food is thought to be one of the most important environmental factors that affect microbial growth throughout all processes between the production and consumption of the food. Generally, the microbial safety of fresh and non-thermally processed foods could be maintained only when the temperature exposed to the food throughout the processes are kept low. However, once the food is exposed to an abusive temperature, microbial contaminants of the food including pathogens and spoilage microbes would grow. Moreover, the increased number of the contaminants does not decrease even when the food is cooled down afterwards. Thus, prediction of microbial growth throughout all the processes from the production to the consumption of the food is very important to food safety and quality.

Furthermore, the temperature of a point in a solid food differs according to its location in the food, and also changes with time. Various types of heat transfer in and around the food are possible. That is, heat conduction might take place in the food. Heat transfer due to vaporization of water from the food surface and heat convection from the air flowing along the periphery of the food is also possible. Thus, it is considerably difficult to estimate the temperature of a point inside the food. Among various types of heat transfer, heat conduction in the food is thought to predominantly determine the temperature of that point. If the surface temperature of the food is known, the complex problem of heat transfer would be reduced to a simple issue of heat conduction. This is because the surface temperatures of the food are the boundary conditions when the heat conduction law is mathematically solved. However, it is generally too difficult to estimate the surface temperature of a food.

In these circumstances, the author aimed to develop a temperature prediction method for food using an unwrapped sausage on a stainless steel tray as a model food (Fujikawa and Kano, 2008). For this, a method for the estimation of the temperature at the surface from measured data was developed.

A refrigerated, commercially available sausage with a cylindrical shape $(50 \mathrm{~mm}$ in diameter and $40 \mathrm{~mm}$ in height) on a rectangular stainless steel tray $(200 \mathrm{~mm}$ $\times 160 \mathrm{~mm} \times 30 \mathrm{~mm}$; $1.5 \mathrm{~mm}$ in thickness) was exposed to a given temperature (Fujikawa and Kano, 2008). The temperatures at defined nodes in the food (Fig. 4) were measured during the exposure. The temperatures of the nodes of the cylindrically-shaped sausage were then estimated with the three-

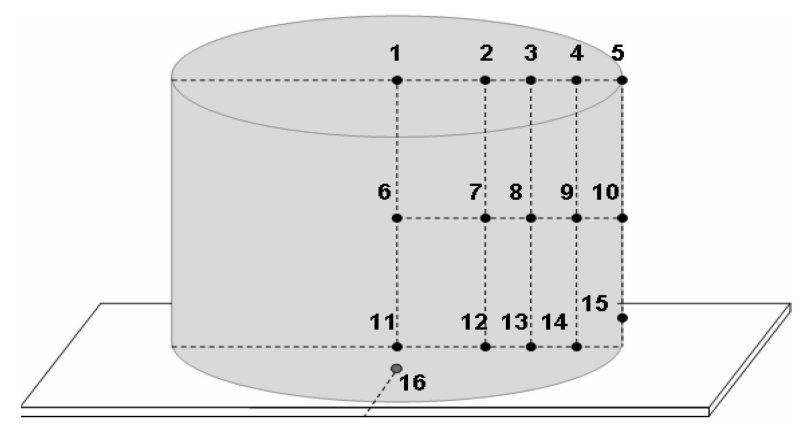

FIG. 4. Nodes constructed in a sausage sample. The numbers correspond to the locations of the nodes at which temperatures are measured. Nodes 1-5, 6-10, and 11-15 were located on the upper surface plane, the middle plane, and the bottom plane, respectively. The distances from nodes 2, 3, 4, and 5 to the center (node 1) on the surface plane were 10, 15, 20, $25 \mathrm{~mm}$, respectively. Node 15 was placed $5 \mathrm{~mm}$ above the bottom plane. 
dimensional heat conduction law (Eq. 5).

$$
\frac{\partial T}{\partial t}=A\left(\frac{\partial^{2} T}{\partial r^{2}}+\frac{1}{r} \frac{\partial T}{\partial r}+\frac{\partial^{2} T}{\partial z^{2}}\right)
$$

Here, $T$ is the temperature $\left({ }^{\circ} \mathrm{C}\right)$ of a node at time $t$ (s), $A$ is the thermal diffusivity of the food $\left(\mathrm{m}^{2} / \mathrm{s}\right)$, and $r$ and $z$ are the radial and $z$-axial coordinates (m), respectively. Eq. 5 was numerically solved with the Crank-Nicolson method, which is unconditionally stable, using a program run on Microsoft Excel (Fuijikawa and Kano, 2008). To solve the equation one needs the initial conditions and the boundary conditions. The former is the temperatures of nodes of the food at the initial time and the latter is those at the surface nodes during the observation period.

The value for $A$, which gives the least square errors between the measured and the estimated temperatures of the nodes, were obtained with the measured temperatures of the nodes. With this value for $A$ and the measured temperatures of the surface nodes in Fig. 4, the temperatures of nodes inside the sausage were successfully predicted with Eq. 5 (data not shown) (Fujikawa and Kano, 2008).

Because using fewer measurement points in this estimation method would be more practical for the food industry, a practical estimation method for temperature was made. That is, two points, the centers of the top and bottom surfaces of a food sample (nodes 1 and 11 in Fig. 4) were selected as standard points for measuring temperatures. When temperatures at the standard nodes and other surface nodes during the exposure at various constant temperatures were measured, the differences between them were not dependent on the temperatures of storage. Thus, with the temperature difference data, the temperatures of surface nodes from measured temperatures of the standard nodes at a given temperature could be estimated. With those estimated surface temperatures, the temperatures of nodes in the food sample were predicted with the heat conduction equation (Eq. 5).

The method successfully predicted temperatures in the sausage sample exposed to constant and dynamic temperatures. At programmed changing temperatures, the method successfully predicted the temperatures of the nodes in the food sample (Fig. 5A, B) (Fuijikawa and Kano, 2008). These results suggest that the method could be a useful tool in predicting the temperature in the food at various patterns of exposure temperature.

The temperature estimation method was further studied with another food sample, a hamburger patty on a stainless steel tray (Fuijikawa and Kano, 2009b). The hamburger patty $(55 \mathrm{~mm} \times 45 \mathrm{~mm} \times$ $15 \mathrm{~mm})$ on the tray $(200 \mathrm{~mm} \times 160 \mathrm{~mm} \times 30 \mathrm{~mm}$;
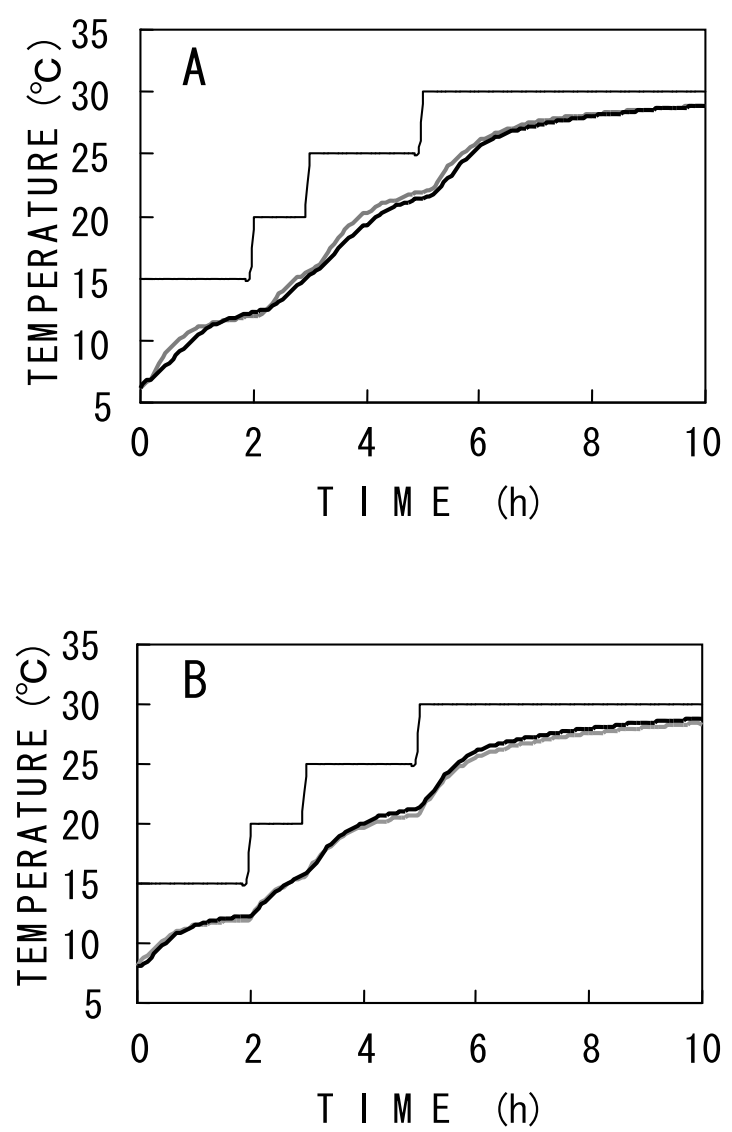

FIG. 5. Temperature prediction in a sausage sample exposed to a dynamic temperature pattern. A step-wise line shows the programmed temperature of storage. Black and gray lines show predicted and measured temperature patterns, respectively, at nodes $6(A)$ and 9 (B) in Fig. 4.

$1.5 \mathrm{~mm}$ in thickness) was defined as a complex of three layers of 5-mm cubes (Fig. 6). The temperatures of the apexes of the cubes making up the patty, referred to as nodes, are estimated using the threedimensional heat conduction law (Eq. 6)

$$
\frac{\partial T}{\partial t}=A\left(\frac{\partial^{2} T}{\partial x^{2}}+\frac{\partial^{2} T}{\partial y^{2}}+\frac{\partial^{2} T}{\partial z^{2}}\right)
$$

where $T$ is the temperature $\left({ }^{\circ} \mathrm{C}\right)$ of a node at time $t$ (s); $A$ is the thermal diffusivity of the food $\left(\mathrm{m}^{2} / \mathrm{s}\right)$; and $x, y$, and $z$ are Cartesian coordinates (m). Eq. 6 was numerically solved with the Crank-Nicolson method.

The temperature estimation method also successfully predicted the temperatures of nodes in the hamburger at constant and dynamic temperatures. Examples at changing storage temperatures are shown in Fig. 7 (Fujikawa and Kano, 2009b). 

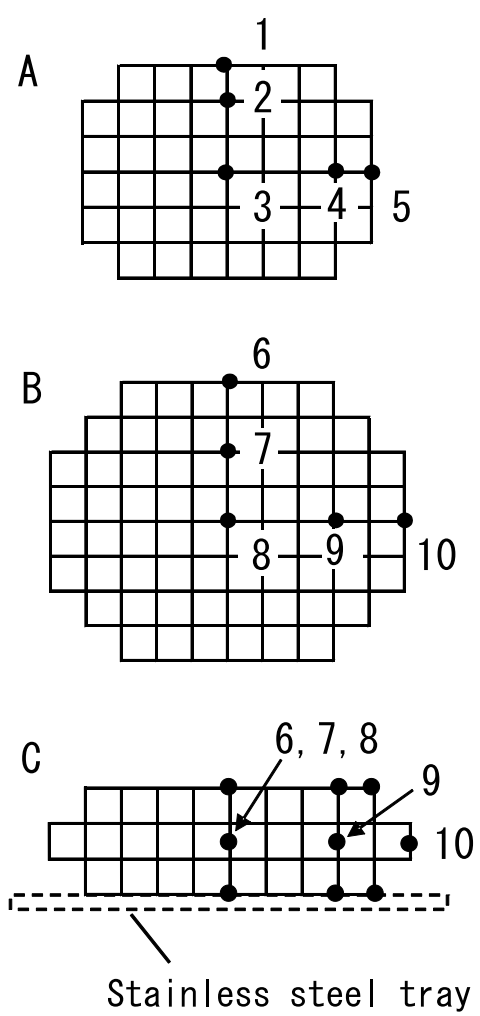

FIG. 6. Three-dimensional image of a hamburger patty. The patty is represented as a complex of $5-\mathrm{mm}$ cubes. A. Top layer of cubes (plane figure). B. Middle layer of cubes (plane figure). C. Sectional figure of the patty. Closed circles represent points at which temperatures are measured by the thermometer. Measured points on the bottom plane (C) are aligned in the same manner as those on the top plane $(A)$. Each measured point is numbered.

\section{B. Simulation of Microbial Growth in Food using its Estimated Temperature}

With the above temperature estimation method for a solid food sample and the new logistic model, a systematic program for predicting microbial growth in the food was developed. On the other hand, in reality, the concentration of microorganisms contaminating a food product may vary according to their location in the food. The estimation system can follow this spatial variation of microbial concentration in a food sample, as shown below.

Suppose that the above hamburger sample is exposed to the storage temperature shown in Fig. 7 and that the food is contaminated with Escherichia coli cells. First, the average temperatures of the cubes located at the centers of the top, middle, and bottom layers of the food (Fig. 6) are estimated (Fig. 8A) (Fujikawa and Kano, 2009b). Here the average temperature of the cube is obtained from the temperatures at the apexes of a cube. Among the cubes at the centers of the top, middle, and bottom layers of
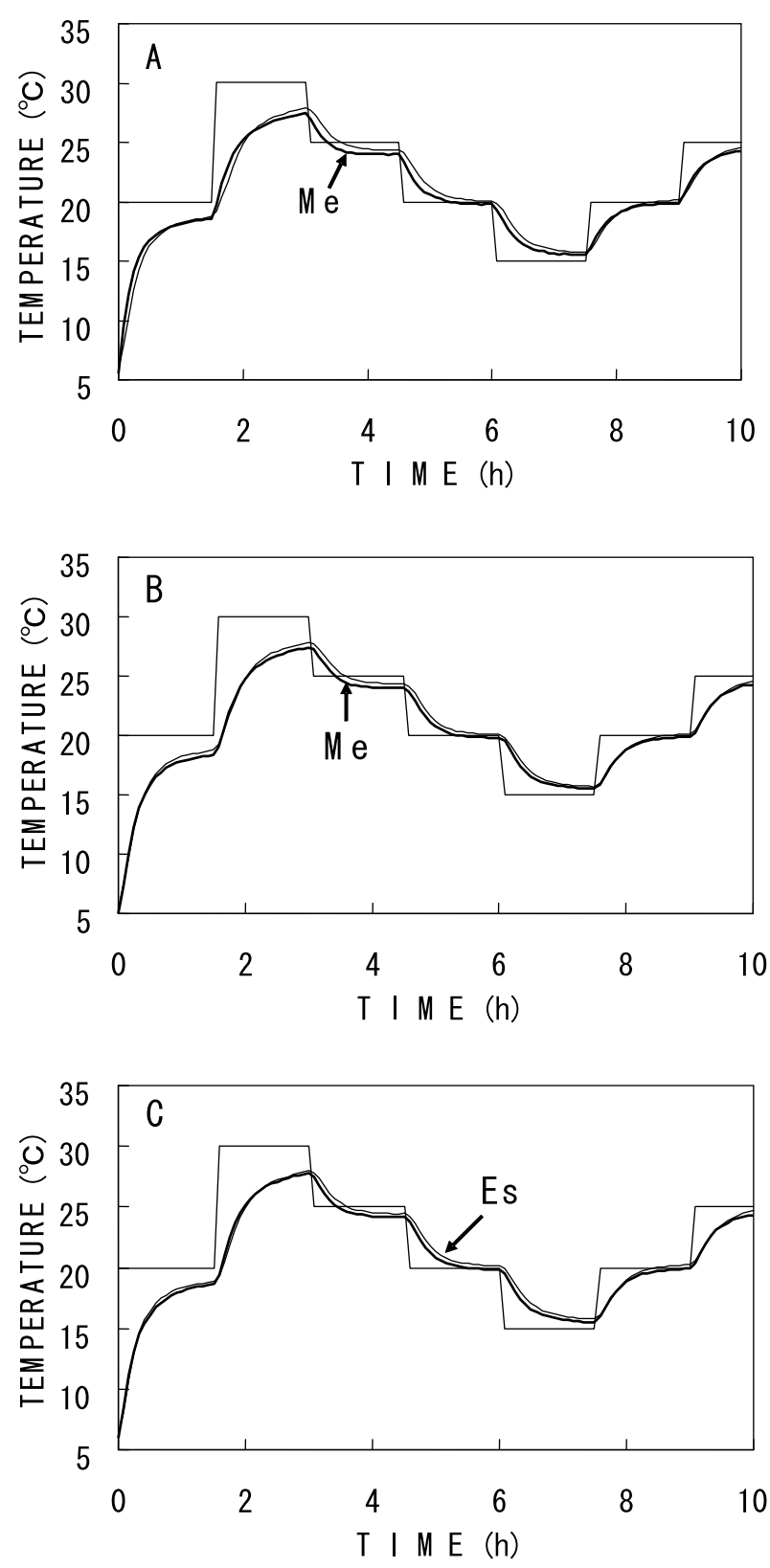

FIG. 7. Temperature estimation for a hamburger patty exposed to changing temperatures. A step-wise line shows the programmed temperature of storage. Thin and thick lines show estimated (Es) and measured (Me) temperature patterns, respectively, at point 7 (A), 8 (B), and 9 (C) in Fig. 6.

the food, the temperature of the bottom cube was the highest (Fig. 8A).

Then bacterial growth in these cubes is predicted with the new logistic model during the exposure (Fujikawa and Kano, 2009b). Parameter values for microbial growth prediction are obtained from experimental data of the author. First, when the food is 

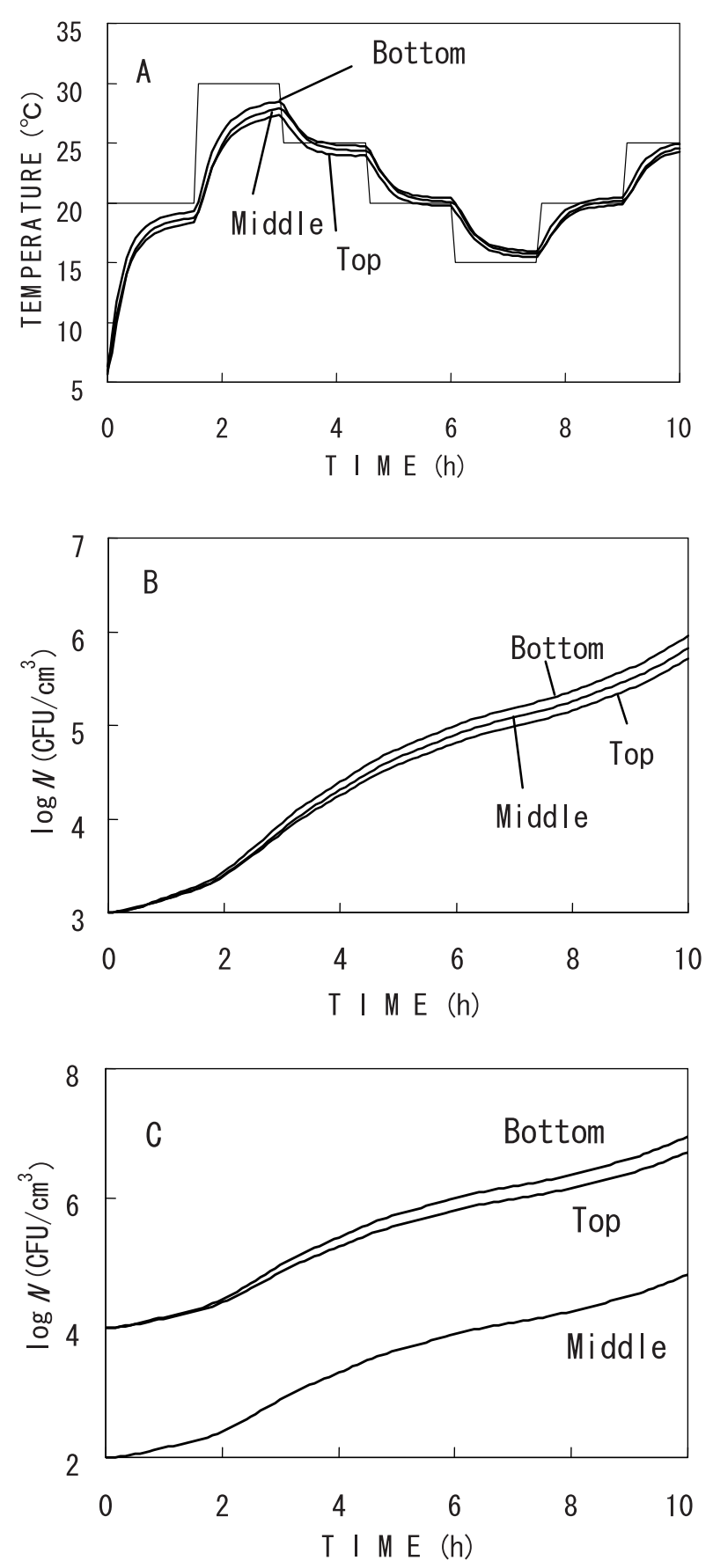

FIG. 8. Bacterial growth prediction in cubes of the hamburger patty exposed to changing temperatures. The food sample was exposed to the temperature pattern shown in Fig. 7. A. Estimated temperature profiles of cubes at the centers of the top, middle, and bottom layers of the food. B. Bacterial growth prediction in the cubes when the food is uniformly contaminated with E. coli cells. C. Bacterial growth prediction in the cubes when the outer cubes of the food which face the atmosphere or the tray (Bottom and Top) are much more heavily contaminated by the microbes than the inner cubes (Middle). uniformly contaminated with E. coli cells at $1 \times 10^{3}$ cell $/ \mathrm{cm}^{3}$, bacterial growth in the cubes is predicted as shown in Fig. 8B. Bacterial growth in the cube at the center of the bottom is the most remarkable (Fig. $8 \mathrm{~B}$ ), because the growth follows temperature (Fig. $8 \mathrm{~A}$ ). The surfaces of most raw foods and food materials are heavily contaminated with microorganisms. When the outer cubes of the hamburger which face the atmosphere or the tray are more heavily contaminated by the organisms $\left(1 \times 10^{4} \mathrm{cell} / \mathrm{cm}^{3}\right)$ compared with the inner cubes $\left(1 \times 10^{2} \mathrm{cell} / \mathrm{cm}^{3}\right)$, bacterial growth for the three cubes is predicted as shown in Fig. 8C.

Few studies have tried to construct a prediction system for heat transfer and microbial growth (Almonacid et al., 2002; Amézquita et al., 2005). Almonacid et al. (2002) simulated Salmonella Enteritidis growth in eggs under various temperature conditions. They thought that the bacteria in the egg would grow exponentially, but actual bacterial growth shows sigmoidal curves. Amézquita et al. (2005) simulated Clostridium perfringens growth during the cooling of cooked boneless ham, but their temperature simulation was two-dimensional.

Microbial growth predicted by our system has not yet been validated by microbiological tests. Further quantitative data on microbial growth in foods are needed. However, the author thinks that the prediction system could be a prototype of an alert system that predicts microbial growth in a given food from its environmental temperature: microbial concentrations of the food of concern predicted by the system could be indices to scientifically judge if the food is microbiologically safe or not. As far as the author knows, an alert system like this has not been developed yet. It could also be a useful tool in the risk assessment of food by giving information on assessment of the exposure during the process from the production to the comsumption of the food.

\section{REFERENCES}

Almonacid, S., Gutierrez, J., Jaques, A., and Simpson, R. (2002). Salmonella Enteritidis risk assessment: a kinetic analysis. J. Food Sci., 67, 1115-1120.

Amézquita, A., Weller, C. L., Wang, L., Thippareddi, H., and Burson, D. E. (2005). Development of an integrated model for heat transfer and dynamic growth of Clostridium perfringens during the cooling of cooked boneless ham. Int. J. Food Microbiol., 101, 123-144.

Baranyi, J. and Roberts, T. A. (1995) Mathematics of predictive food microbiology. Int. J. Food Microbiol., 26, 199-218.

Buchanan, R. L., Whiting, R. C., and Damert, W.C. (1997) When is simple good enough: a comparison of the Gompertz, Baranyi, and three-phase linear models for 
fitting bacterial growth curves. Food Microbiol., 14, 313326.

Fujikawa, H., Kai, A., and Morozumi, S. (2003) A new logistic model for bacterial growth. J. Food Hyg. Soc. Japan, 44, 155-160.

Fujikawa, H. and Morozumi, S. (2005) Modeling surface growth of Escherichia coli on agar plates. Appl. Environ. Microbiol., 71, 7920-7926.

Fujikawa, H., Yano, K., Morozumi, S., Kimura, B., and Fujii, T. (2006) Development of a predictive program for microbial growth under various temperature conditions. (in Japanese) J. Food Hyg. Soc. Japan, 47, 288-292.

Fujikawa, H. and Morozumi S. (2006) Modeling Staphylococcus aureus growth and enterotoxin production in milk. Food Microbiol., 23, 260-267.

Fujikawa, H. and Kano, Y. (2008) A new method for estimation of temperature in food exposed to abuse temperature. Food Sci. Technol. Res., 14, 111-116.

Fujikawa, H. and Kano, Y. (2009a) Development of a program to fit data to a new logistic model for microbial growth. Biocont. Sci., 14, 83-86.
Fujikawa, H. and Kano, Y. (2009b) Simulation of microbial growth in food using its estimated temperature. Food Sci. Technol. Res., 15, 127-132.

Fujikawa, H., Kimura, B., and Fujii, T. (2009) Development of a predictive program for Vibrio paraphaemolyticus growth under various environmental conditions. Biocont. Sci., 14, 127-131.

Fujikawa, H. (2010) Development of a new logistic model for microbial growth in foods. Biocont. Sci., 15, 75-80.

Gibson, A. M., Bratchell, N., and Roberts, T. A. (1987) The effect of sodium chloride and temperature on the rate and extent of growth of Clostridium botulinum type $\mathrm{A}$ in pasteurized pork slurry. J. Appl. Bacteriol., 62, 479-490.

Kimura, B., Sekine, Y., Takahashi, H., Tanaka, Y., Obata, H., Kai, A., Morozumi, S., and Fujii, T. (2008) Multiple-locus variable-number of tandem-repeats analysis distinguishes Vibrio parahaemolyticus pandemic O3:K6 strains. J. Microbiol. Meth., 72, 313-320.

McMeekin, T. A., Olley, J. N., Ross, T., and Ratkowsky, D. A. (1993) Predictive Microbiology: Theory and Application. Research Studies Press, Taunton (UK). 